\section{La Universidad escucha a las escuelas: balance de una acción sinérgica}

\section{Gabriela Rotondi}

Docente investigadora de la Facultad

de Ciencias Sociales, Universidad

Nacional de Córdoba, Argentina.

gabrielarotondi@hotmail.com
Integración de la docencia y la extensión /

Intervenciones

RECEPCIÓN: 24/06/16

ACEPTACIÓN FINAL: 10/10/16

\section{Resumen}

El presente trabajo se plantea el desafío de trasmitir una experiencia que se define desde sus fundamentos como sinérgica. Se trata de las apuestas extensionistas de la cátedra Teoría, Espacios y Estrategias de intervención IV - institucional, correspondiente a la Licenciatura en Trabajo Social, de la Facultad de Ciencias Sociales de la Universidad Nacional de Córdoba. Intentaremos dar cuenta de algunos de los vínculos planteados entre las tres funciones universitarias (docencia, investigación y extensión) y su presencia en la instancia de cátedra, como núcleo básico de la acción entre docentes y estudiantes. Tomaremos el período desarrollado desde 2001-2016, período en el cual el trabajo extensionista se institucionaliza a partir de una propuesta, el Programa "La Universidad escucha las escuelas", que toma cuerpo a partir de la hiperinflación de 2001, momento en que la cátedra lo fundamenta y diseña en el marco de un contexto que instala un fuerte cambio en las escuelas públicas, respecto de sus servicios sociales a la comunidad.

Palabras-clave

- Universidad

- Escuelas

- Sinergia

- Funciones

\section{Resumo}

O presente trabalho se propõe o desafio de transmitir uma experiência que se define a partir dos seus fundamentos como sinérgica. Trata-se das apostas extensionistas da cadeira Teoria, Espaços e Estratégias de Intervenção IV correspondente ao Bacharelado em Serviço Social, da Faculdade de Ciências Sociais da Universidade Nacional de Córdoba. Tentaremos explicar alguns dos vínculos criados entre as três funções da universidade (ensino, pesquisa e extensão) e sua presença na instância de ensino, como núcleo básico da ação entre professores e estudantes. Nós tomaremos o trajeto do período de 2001-2016, durante o qual o trabalho de extensão é institucionalizado a partir de uma proposta, o programa "A Universidade escuta as escolas", que toma forma a partir da hiperinflação de 2001, quando a cadeira o fundamenta e o planeja dentro de um contexto que instala uma grande mudança nas escolas públicas, a respeito dos seus serviços sociais para a comunidade.

Palavras-chave

- Universidade

- Escolas

- Sinergia

- Funções

Para citación de este artículo

Rotondi, G. (2016). La Universidad escucha a las escuelas: balance de una acción sinérgica. En Revista +E versión digital, (6), pp. 382-387. Santa Fe, Argentina: Ediciones UNL. 


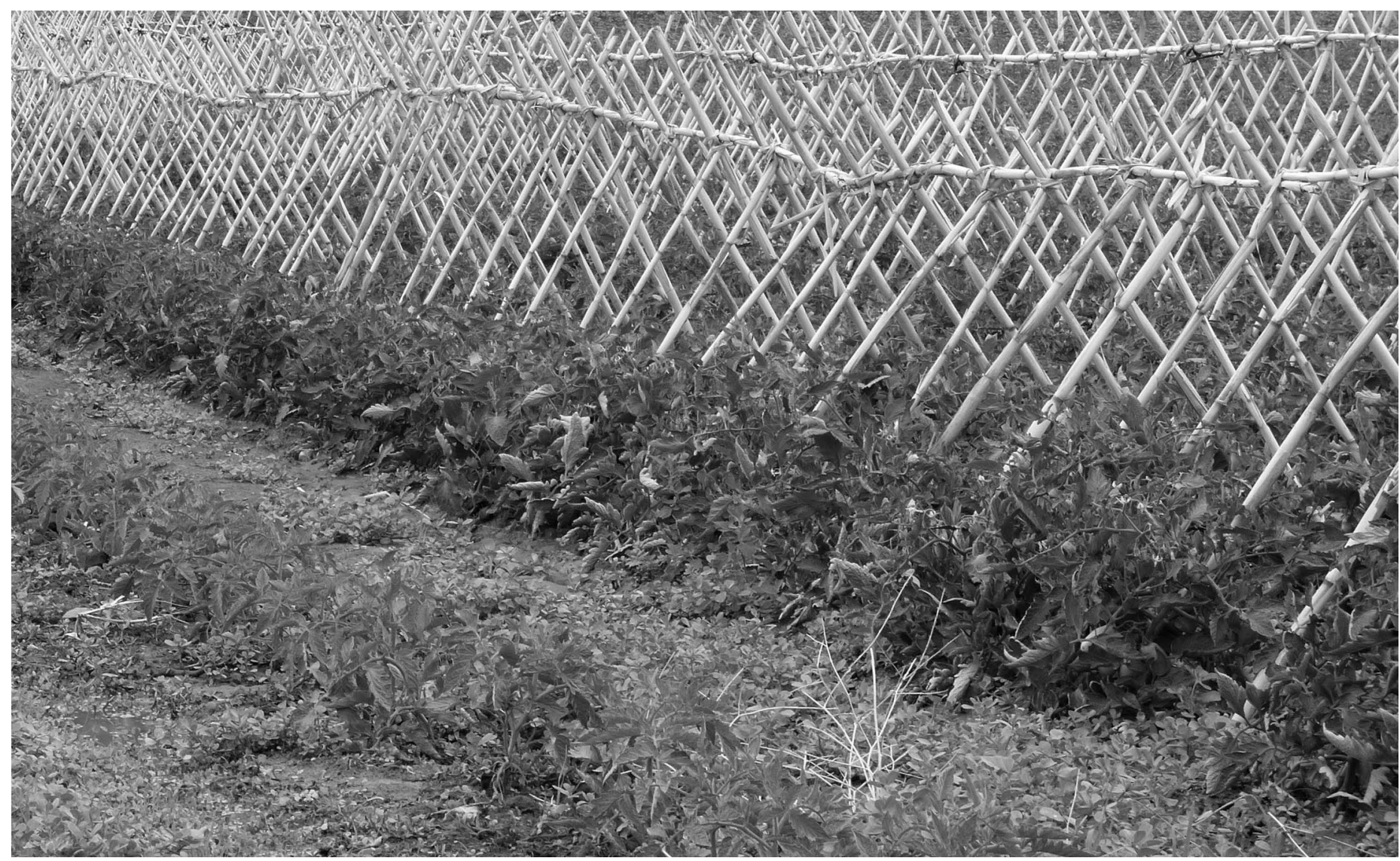

(C) Block Micaela

\section{Introducción}

El presente trabajo se plantea el desafío de trasmitir una experiencia que se define desde sus fundamentos como sinérgica. Se trata de las apuestas extensionistas de la cátedra Teoría, Espacios y Estrategias de intervención IV - institucional (en adelante TEEI IV), correspondiente a la Licenciatura en Trabajo Social, de la Facultad de Ciencias Sociales de la Universidad Nacional de Córdoba y su equivalente, Trabajo Social V - Intervención Institucional denominada así hasta 2004. Intentaremos dar cuenta de algunos de los vínculos planteados entre las tres funciones universitarias (docencia, investigación y extensión) y sus expresiones en la instancia de cátedra, en tanto núcleo básico de la acción entre docentes y estudiantes.

Decimos trabajar desde las tres funciones universitarias, la docencia, la extensión y la investigación, ya definidas en la trama curricular de la Licenciatura en Trabajo Social, pero demás planteadas a

1) La propuesta de "educación en servicio" en el marco de la cátedra TEEI IV - Instituciones, plantea la oportunidad de dejar instaladas situaciones de aprendizaje a partir de una acción que se lleva adelante articulando la práctica académica de la materia (centrada en intervención en instituciones desde demandas genuinas) y las posibilidades que dicha experiencia

partir de la posición y la toma de posición del equipo docente. Tomaremos en particular el período desarrollado desde 2001 y hasta 2016, período en el cual el trabajo extensionista se institucionaliza a partir de una propuesta de "educación en servicio"1 en el marco de la cátedra mencionada en el Programa "La Universidad escucha las escuelas" 2 . El mismo toma cuerpo a partir de la hiperinflación de 2001, momento en que la cátedra lo fundamenta y diseña además como programa de extensión y de formación de estudiantes, esto planteado en el marco de un contexto que instala un fuerte cambio en las escuelas públicas respecto de sus servicios sociales a la comunidad. En ese momento, la escuela pública, se ve obligada a aportar, con mayor énfasis, en una necesidad básica de sus estudiantes, la alimentación, esto a través de los comedores que se instalan masivamente en las escuelas. Esta situación trae aparejadas diversas conflictivas e interrogantes tanto a las instituciones como a sus sujetos/as.

permite a partir del servicio que llevan adelante los estudiantes y docentes en las instituciones aportando desde el ejercicio preprofesional. Se construyen experiencias y se aprende de ellas.
2) A 15 años del Programa "La Universidad escucha a las escuelas" Escuela de Trabajo Social, hoy Facultad de Ciencias Sociales, Universidad Nacional de Córdoba. 
Esta cátedra que ofrece estudios y servicios concretos a instituciones y organizaciones decide enfatizar su aporte a las escuelas y hace su propuesta teniendo en cuenta el contexto social y político de las escuelas de Córdoba, los recursos propios relativos al conocimiento de instituciones y organizaciones, sus recursos docentes y estudiantiles y una toma de posición ante un sector como la escuela pública, que se ve interpelado por la compleja realidad de los estudiantes.

Desde lo académico, y dado que la cátedra se centra en el estudio y entrenamiento de la intervención social en torno a las situaciones de malestar, conflicto y crisis institucional desde una disciplina específica, el Trabajo Social, el equipo se propone: aportar a la comunidad con sus prácticas académico-extensionistas, asumiendo como prioritarias (a nivel de espacios de práctica preprofesional) las escuelas públicas, ubicadas en barrios populares, de diversos niveles. Allí se centran los esfuerzos teóricos y prácticos de la cátedra. La misma, perteneciente al $4^{\circ}$ año del plan de estudios de la Licenciatura en Trabajo Social, realiza acciones de docencia y extensión, y posteriormente el equipo, recién desde 2004, asienta en la misma área sus esfuerzos de investigación, año donde además nos encontramos ante el desafío de la implementación de un nuevo plan de estudios de la carrera. Presentaremos entonces en este trabajo algunas de las líneas y rasgos de un proceso que buscó desde su origen la sinergia de funciones y que presentó diversas instancias de acción directa en la escena institucional, algunos logros y también dificultades presentes en una tarea que fuera diseñada desde las tres funciones.

\section{Iniciando a través de la demanda social}

Una primera cuestión considerada a nivel de la construcción del programa, y que sin duda encuentra relación con la disciplina, Trabajo Social, fue el reconocimiento de los espacios universitarios como espacios de funciones múltiples, donde la extensión es parte en una trama construida ya desde la visión curricular que tiene quien diseña. La tarea troncal de la carrera es realizada por materias teórico-prácticas (denominadas Teoría, espacios y estrategias e intervención I, I, II y IV), cuestión que aporta elementos para una mirada que trata de integrar y concebir la formación con los diversos componentes, y que se lleva adelante durante los diversos años de la carrera.

Se busca la mirada de un escenario particular, que en este caso son las instituciones y organizaciones (así como en otros es la comunidad y la familia), y se asumen prioritariamente las escuelas en este caso, en consideración que opera como contexto la pertenencia a un mismo sector de la educación que estaba en un momento de crisis. Trabajamos con relación a un sujeto social particular (los sectores populares) pero también particulares habitantes de las instituciones educativas (estudiantes, docentes, no docentes y directivos) y abordamos un objeto de intervención con diversas aristas (aquello que desde el Trabajo Social plantea abordar las necesidades, las relaciones sociales y las representaciones sociales).

En este contexto, el Programa "La Universidad escucha las escuelas" se lleva adelante en el marco de una cátedra pero plantea sus propios objetivos, y por ello realizamos el recorte, atendiendo a que está centrado en capacitación, intervención e implementación de políticas y redes. Los objetivos del Programa son los siguientes:

"1. Desarrollar servicios académicos y extensionistas desde procesos de intervención institucional en escuelas públicas de Córdoba (capital e interior) vinculados al abordaje de problemáticas sociales que se instalan en el espacio educativo y que son factibles de abordar desde el Trabajo Social.

2. Desarrollar proyectos de investigación vinculados a la realidad educativa y las problemáticas sociales presentes en la escuela.

3. Facilitar procesos de reflexión y transferencias de investigación en las escuelas públicas, involucrando los diversos actores institucionales a los fines de desarrollar estrategias de articulación escuela/universidad para resolver problemáticas concretas incidiendo conjuntamente en políticas públicas.

4. Producir herramientas para el abordaje de problemas sociales en las escuelas que facilitan los aportes universitarios y permitan la construcción conjunta de materiales entre los actores universitarios y los actores participantes de las escuelas". Programa La Universidad escucha las escuelas." (Rotondi y otros, 2015:3)

Posibilitar la sinergia de las tres funciones de docencia, investigación y extensión, tiene relación no solo con la posición de quienes conducen, los docentes, sino además con el posicionamiento de los actores universitarios y con las definiciones de la trama curricular tanto en su concepción, como en sus espacios de acción concreta. La cátedra TEEI IV, que en ese momento se denominaba Trabajo Social $\mathrm{V},{ }^{3}$ se plantea un proceso teórico con práctica académica-extensionista obligatoria para los estudiantes, tal como indica el plan de estudios, en la cual se plantea la instrumentación con relación a uno de los niveles de abordaje de la disciplina, el institucional-organizacional. ${ }^{4} \mathrm{Y}$ si bien el equipo en aquellos
3) El actual Plan de estudios de la Licenciatura en Trabajo Social, data de 2004, y las acciones en escuelas públicas con mayor dedicación se inician en 2001.
4) A lo largo de la carrera Licenciatura en Trabajo Social, las materias troncales plantean la condición de ser teórico-prácticas. En el caso de la ma- teria TEEI IV y dado que se encuentra en el $4^{\circ}$ año de la carrera, la posibilidad de habilitar un espacio teórico-práctico de por sí aporta una particular sinergia que se ve enriquecida con las líneas de investigación del equipo y con la mirada de la perspectiva extensionista de dichas prácticas. 
años aún no contaba con investigaciones propias de la escena institucional, algunos miembros veníamos desarrollando procesos de investigación en torno a los valores y las prácticas de la ciudadanía cordobesa, y la vigencia de los valores ciudadanos en Córdoba, así como de la cuestión que establecía vínculos entre la ciudadanía y sus relaciones con la intervención social. ${ }^{5}$

Por otra parte, la lectura del contexto y la identificación de la demanda social hacia la Universidad pública fueron otros aspectos que permitieron objetivar las cuestiones, diseñar un programa y encontrarnos con los sujetos que plantean y especifican problemáticas a partir de una modalidad que instala la cátedra en cuanto a la viabilidad del ingreso de estudiantes a las instituciones. Ya que en todos los casos, y desde 2001 más precisamente, se ingresa a los espacios de escuelas, guarderías, clubes, centros de jubilados, etc., "únicamente ante la demanda de las instituciones", ante una convocatoria concreta de los miembros de las organizaciones, que viven y señalan algún tipo de malestar, conflicto y/o crisis institucional. Por otra parte, la ubicación de la demanda social en un sector específico, como los sectores populares de Córdoba plantea un acercamiento a una realidad barrial en la cual se asientan el grueso de las instituciones educativas del sector público, y en torno al cual se define la intervención desde el programa mencionado.

Esta situación de trabajo solamente ante casos de demanda social permanece hasta la fecha con el Programa y constituye además el antecedente de las prácticas de investigación y acción del equipo (que se inician en 2004 y continúan hasta el momento). El espacio del programa es sin duda el ámbito desde el cual se generan las preguntas de investigación respecto de las demandas abordadas y estudiadas.

"Dicho Programa, dirigido a las escuelas públicas que se encuentren atravesando situaciones de conflicto, crisis y/o malestar, y que requieran ser atendidas desde Trabajo Social, permite, bajo una modalidad de aprendizaje-servicio, establecer un acuerdo de intervención en torno a diversas problemáticas, malestares y conflictos institucionales entre los actores que habitan la misma. Sobre esa experiencia se formulan interrogantes que, posteriormente, dan sentido a la presente investigación.

Sin ánimo de agotar aquí las preguntas que nos surgieron, transcribimos algunas de ellas:

¿Son las instituciones educativas espacios abiertos a las políticas sociales? ¿Qué respuestas puede aportar el trabajo social a las múltiples demandas que plantean las Instituciones?
¿Qué desplazamientos provoca 'lo social' en lo Institucional que requieran de la intervención del trabajo social? ¿Cómo se construyen las necesidades sociales en el escenario institucional?". (Rotondi y otros, 2006:171-172)

El proceso del seguimiento de la demanda social constituye un elemento que articula el plan de trabajo de la cátedra a una línea concreta de docencia, extensión e investigación y consolida líneas en los tres casos. Y los proyectos de extensión que se desarrollan desde la cátedra presentan ese rasgo integrador de articulación defunciones. Un ejemplo de ello que consolida de manera significativa la línea de extensión del equipo fue el Programa "Universidad y Escuela Pública: abordar la violencia para promover inclusión social”, el cual fue premiado en el Concurso Nacional del Programa de mejora de la escuela media del Ministerio de Educación de la Nación. El Programa fue llevado adelante entre 2005 y 2007 con fondos propios, permitiendo realizar procesos de evaluación y facilitar la acción directa del equipo, así como elaborar producciones permanentes de artículos, textos, e inclusive libros y DVD que circulan y retroalimentan los procesos. Estas tareas, en conjunto con la investigación de la cátedra (iniciada en 2004 sobre la misma temática), permite que el equipo realice un mapeo que facilitó decisiones extensionistas y de investigación. Por otra parte, el hecho de trabajar colectivamente sobre la base del equipo de cátedra facilitó la articulación de un colectivo que emprendía y accionaba sobre una estructura que la misma Escuela de Trabajo Social sostenía desde su estructura.

En ese momento, sobre una casuística de 30 demandas, se identificaron seis problemáticas específicas que expresaban demandas que la escuela pública planteaba para la intervención social a la cátedra y que se expusieron en una producción de la cátedra que se compiló y publicó en 2008. Las demandas se identifican como (Rotondi, 2008):

- Convivencia escolar y violencia en la escuela.

- Dificultades organizativas.

- Problemas de autoritarismo.

- Reformulación de proyectos institucionales.

- Convivencia y prevención de conductas agresivas y maltrato.

- Fortalecimiento de redes sociales de apoyo a las escuelas.

Sobre estas demandas, la continuidad de la extensión universitaria articulada en el marco de la cátedra a docencia e investigación se profundiza y fortalece con otros proyectos que fueron buscados y gestionados a tal fin. Así, el proyecto "Hacia la equidad de género
5) En mi caso personal participé en los proyectos dirigidos por Nora Aquín entre 1998 y 2003 , estudios que son un claro aporte tanto a la disciplina, como a mis particulares trayectos, lo cual realmente agradezco. 
en el sistema educativo", que fuera premiado por la Facultad Latinoamericana de Ciencias Sociales (FLACSO) en el Fondo concursable en 2007 e implementado entre 2007-2008 con foco en investigación y que ofrece aperturas del asunto hacia otras cátedras. El proyecto "Universidad y Escuela Pública: conflictividad en la escuela, debates y abordajes", que se desarrolla dentro del Programa de Transferencia de Resultados de la Investigación del Ministerio de Ciencia y Tecnología de la Provincia de Córdoba implementado entre 2008-2009 y que facilita la llegada de lo investigado a las instituciones que referencian el conocimiento. Y más tarde, el proyecto "Agremiación y Acción juvenil: un derecho ciudadano que forma ciudadanos/as (2009-2010)", y "Agremiación y Acción Juvenil (2012-2013)", ambos premiados por el Programa Nacional de Voluntariado Universitario en la Convocatoria 2012 y que claramente facilitan la llegada a los sujetos con quienes se comparte la tarea al interior de la escuela, básicamente estudiantes secundarios.

Para el equipo resulta claro que sin la sincronía de las tres funciones estas experiencias no hubieran sido posibles ni viables las acciones. Los proyectos y las acciones se retroalimentan en diálogo con los actores de las instituciones que acompañan a un equipo de conformación múltiple: docentes, estudiantes y egresados desde la Universidad y docentes, estudiantes y personal administrativo desde las escuelas.

Los proyectos de investigación de la cátedra (entre 2004 y hasta la fecha) plantean vínculos con aquellas situaciones, demandas y problemáticas que se expresan en escenarios de intervención concretos y hacia los cuales van dirigidas las estrategias $y$ acciones de estudiantes y docentes. Por otra parte, las acciones intervenidas se proyectan como conjuntas con las instituciones que demandan pero que además reclaman la devolución del conocimiento producido.

\section{Funciones y misiones. La extensión en el curriculum}

Debemos señalar que el equipo que lleva adelante la tarea, plantea varios movimientos, propios de la cátedra en sí, diversos docentes circulan por el espacio y aportan visiones, e intervenciones concretas. La mirada va articulando funciones y estableciendo nexos entre las prácticas docentes y los proyectos extensionistas que se van creando y desarrollando. En tal sentido, cabe mencionar además que la mirada de problemas sociales particulares de las instituciones es un punto clave en la cuestión pero también la consideración de la teoría, los espacios y las estrategias a la hora de intervenir en instituciones. El nivel de abordaje en instituciones y organizaciones desde el Trabajo Social es el más reciente y debemos ubicarlo, en nuestra escuela al menos, a partir de los años ' 90 . Estas prácticas se van particularizando en esa década y presentan problemáticas que se hacen factibles de ser intervenidos desde el Trabajo Social institucional en un espacio específico.
Se van involucrando y desarrollando desde la docencia y también desde extensión de la cátedra, y se van respaldando desde el abordaje docente problemas concretos, de las instituciones. Tal el caso, por ejemplo, de la educación sexual integral en la escuela; la violencia en las instituciones, el trabajo sobre violencia en el noviazgo y agremiación juvenil, o las cuestiones ambientales que van permitiendo y dando desarrollo a las acciones concretas. Intervienen el equipo docente y los estudiantes; se investiga y se extiende de manera colectiva, sumando ayudantes motivados por la política sinérgica de la cátedra. Las miradas que se llevan adelante permiten redefinir funciones y misiones institucionales.

Gráfico $N^{\circ} 1$. Casuística de las demandas atendidas por el equipo entre 2001 y 2014. Sistematización de la Información cátedra TEEI IV

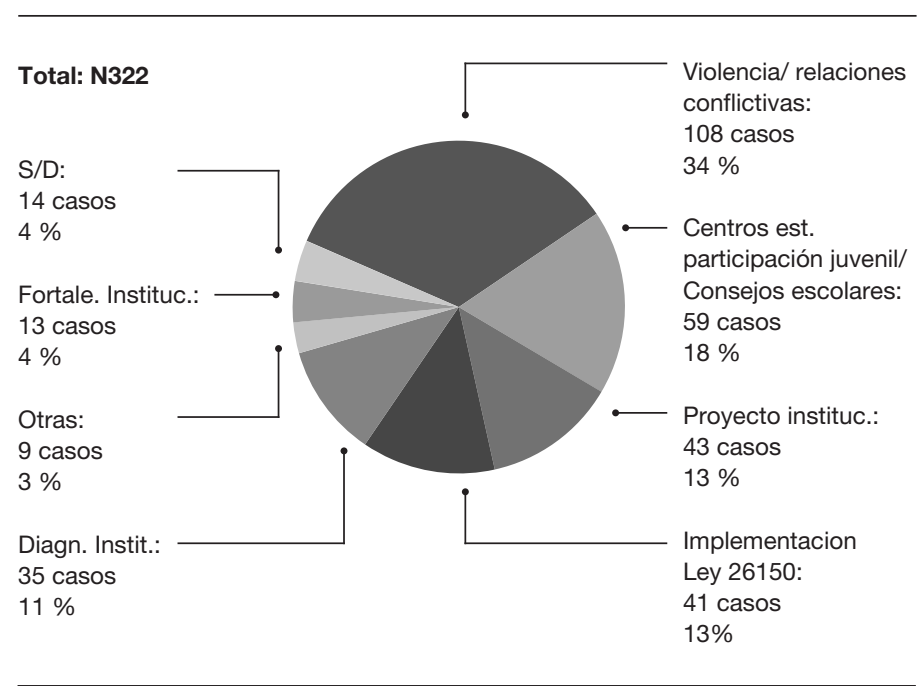

Fuente: Verón (2015)

Las estrategias y acciones desarrolladas hasta el momento en el marco de la cátedra se han planteado en torno a las problemáticas señaladas en el Gráfico $N^{\circ} 1$ pero atendiendo a líneas de acción de las cuales resumimos aquí las más frecuentes y de manera acotada por motivos de espacio:

1) Líneas de acción con foco en la capacitación de los actores institucionales de la escuela pública (docentes, directivos, estudiantes y preceptores), donde las definiciones de los focos se realiza en el marco de la actividad compartida y los diagnósticos desarrollados por las profesionales.

2) Intervención social en situaciones de malestar, conflicto y crisis institucionales relacionadas con la violencia institucional, y la construcción de proyectos institucionales, cuestiones que se llevan adelante a través de "demandas concretas de actores institucionales".

3) Implementación, seguimiento y crítica de políticas públicas particulares, por ejemplo aquellas que se relacionan con la Ley 26150 de Educación sexual integral, en cuyo caso se abordan 
las problemáticas de la violencia en el noviazgo, la agremiación juvenil, problemas organizativos y las problemáticas ambientales. 4) Construcción de redes y acción en el marco comunitario de las instituciones, buscando encuentros, acuerdos y proyección de estrategias comunes que aporten a la continuidad y el sostenimiento. 5) Producción y devolución de conocimiento mediante materiales que se desarrollan con insumos de las investigaciones asociadas al proyecto, y de la recuperación de los procesos de intervención desarrollados, buscando incorporar la palabra de los/las diversos/ as actores sociales.

Estas líneas demandan una posición del equipo, de los docentes, de la cátedra, por lo que dejo expresado en el siguiente punto el posicionamiento impulsado.

\section{Posición y posicionamiento}

Un aspecto a considerar en este punto es el hecho de concebir la extensión como parte del insumo académico y las propias prácticas académicas como extensionistas. Las relaciones permanentes, los insumos en diálogo son claramente aristas de la cuestión que permite y fortalece las prácticas concretas, así como la llegada a terreno con los estudiantes. Sin embargo, no siempre los docentes pueden dar desarrollo a su tarea desde ese ángulo, y cuestiones como la masividad y la burocracia a la hora de los acuerdos y convenios con las instituciones son elementos que juegan respecto de la sinergia de las tres funciones.

Otro aspecto que debemos considerar es el hecho de asumir a los actores que se vinculan a la extensión universitaria como los primeros receptores de las transferencias de investigación. Pedimos palabra, interrogamos y debatimos, pero la palabra se devuelve en un diálogo que facilita el crecimiento de los diversos sujetos, siendo el equipo universitario un sujeto más de prácticas compartidas. Diálogo y permanencia/continuidad son claves del asunto, y debemos señalar también la búsqueda de institucionalidad y reconocimiento de lo que se realiza. Mediante el Programa "La Universidad escucha las escuelas" pretendimos establecer un puente entre la Universidad y la sociedad a través de propuestas concretas. Consideramos que desde allí se puede desarrollar una política de extensión con alternativas concretas para que se indaguen, traten y aborden problemas sociales en articulación con otros actores sociales. La tarea se realiza desde tres aristas que plantean ya en su acción su propia sinergia: la docencia (particularmente en lo relativo al plan de prácticas académicas del cuarto nivel), la extensión que llevan adelante docentes y ayudantes junto a las transferencias de investigación, y la investigación que ha permitido desarrollar conocimientos articulados al programa desde diversos proyectos de investigación del equipo.

Una clave tal vez que aún no hemos mencionado es el hecho de que el equipo docente hace convocatorias públicas para recibir las demandas de las escuelas. Las escuelas plantean sus demandas convocadas por los medios masivos universitarios que hacen lo que denominamos "convocatoria pública a escuelas" que deseen que el equipo de cátedra aporte al abordaje de situaciones de malestar, conflicto y crisis institucional. Esta convocatoria ha recibido de manera sistemática y anualmente demandas de las escuelas públicas en un número que se acerca, desde 2001 y hasta la fecha, a más de 400 . De las mismas, se dividen aquellas que serán abordadas por alumnos en su prácticas académicoextensionistas y aquellas que abordará el equipo docente junto a los adscriptos y ayudantes, con relación a la extensión y/o investigación o articuladas entre las diversas funciones. Se articulan además a este Programa los proyectos de becarios extensionistas interdisciplinarios (que concursan y llegan a pertenecer al Programa "Becas de Extensión Universitarias"). La permanencia del Programa "La Universidad escucha a las escuelas" ha permitido que los resultados de los proyectos de investigación que se llevan adelante sean claramente transferidos de manera sistemática a escuelas y espacios de la política pública educativa por el mismo equipo. Este hecho, el diálogo, es tal vez lo que ha permitido que se generen emprendimientos netamente extensionistas que han ido obteniendo sus propios fondos para la tarea y para la producción de sus transferencias mediante libros, textos y material digital diverso.

\section{Referencias bibliográficas}

Rotondi, G. (2008). Universidad y escuela pública: abordar la violencia para promover la inclusión social. Escuela de Trabajo Social. Espartaco Editorial.

Rotondi, G. (2016). Programa Cátedra Teoría, espacios y estrategias de intervención institucional, UNC (2016). Programa "La Universidad escucha a las escuelas" Escuela de Trabajo Social, hoy Facultad de Ciencias Sociales, Universidad Nacional de Córdoba.

Rotondi, G.; Fonseca, C. y Verón, D. (2006). Necesidades que plantean las demandas de intervención institucional en escuelas "al" trabajo social. En Aquín, N. (Comp.), Reconstruyendo lo social. Prácticas y experiencias de investigación en Trabajo Social (pp. 171-190). Buenos Aires: Espacio Editorial.

Verón, D. (2015). Sistematización de la Información Cátedra TEEI IV. Casuística de las demandas atendidas por el equipo entre 2001 y 2014. 